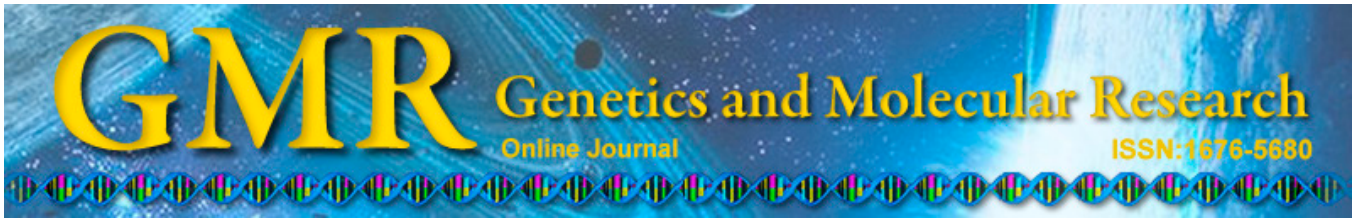

\title{
Ascorbate peroxidase from Jatropha curcas enhances salt tolerance in transgenic Arabidopsis
}

\author{
Y. Chen' ${ }^{1}$, J. Cai' ${ }^{2}$, F.X.Yang ${ }^{3}$, B. Zhou ${ }^{1}$ and L.R. Zhou ${ }^{1}$ \\ ${ }^{1}$ College of Architecture and Environment, Sichuan University, Chengdu, \\ Sichuan, China \\ ${ }^{2}$ West China School of Pharmacy, Sichuan University, Chengdu, Sichuan, China \\ ${ }^{3}$ Guangdong Key Laboratory of Ornamental Plant Germplasm Innovation and \\ Utilization, Environmental Horticulture Research Institute, \\ Guangdong Academy of Agricultural Sciences, Guangzhou, Guangdong, China
}

Corresponding author: L.R. Zhou

E-mail: zhoulr@mail.ubc.ca

Genet. Mol. Res. 14 (2): 4879-4889 (2015)

Received July 10, 2014

Accepted November 26, 2014

Published May 11, 2015

DOI http://dx.doi.org/10.4238/2015.May.11.20

\begin{abstract}
Ascorbate peroxidase (APX) plays a central role in the ascorbate-glutathione cycle and is a key enzyme in cellular $\mathrm{H}_{2} \mathrm{O}_{2}$ metabolism. It includes a family of isoenzymes with different characteristics, which are identified in many higher plants. In the present study, we isolated the $A P X$ gene from Jatropha curcas $L$, which is similar with other previously characterized APXs as revealed by alignment and phylogenetic analysis of its deduced amino acid sequence. Real-time qPCR analysis showed that the expression level of JcAPX transcript significantly increased under $\mathrm{NaCl}$ stress. Subsequently, to elucidate the contribution of $J C A P X$ to the protection against salt-induced oxidative stress, the expression construct $p 35 S$ : JcAPX was created and transformed into Arabidopsis and transcribed. Under 150-mM NaCl stress, compared with wild type (WT), the overexpression of $J c A P X$ in Arabidopsis increased the germination rate, the number of leaves,
\end{abstract}


and the rosette area. In addition, the transgenic plants had longer roots, higher total chlorophyll content, higher total APX activity, and lower $\mathrm{H}_{2} \mathrm{O}_{2}$ content than the WT under $\mathrm{NaCl}$ stress conditions. These results suggested that higher APX activity in transgenic lines increases the salt tolerance by enhancing scavenging capacity for reactive oxygen species under $\mathrm{NaCl}$ stress conditions.

Key words: Oxidative stress; Ascorbate peroxidase; Salt stress; $\mathrm{H}_{2} \mathrm{O}_{2}$; Jatropha curcas

\section{INTRODUCTION}

Soil salinity is one of the most important environmental stress factors that significantly decreases the yield of a wide variety of crops worldwide (Zhu, 2001). Salt stress may alter biochemical pathways and physiological responses mediated by an increased generation of reactive oxygen species (ROS) (Zhu et al., 2013). Accumulation of high levels of ROS perturbs or overwhelms anti-oxidative defense, which could lead to severe damages such as lipid peroxidation, protein denaturation, and DNA mutation (Imlay, 2008). To prevent damage to cellular components by ROS, plants have developed a complex antioxidant system comprised of non-enzymatic antioxidants such as ascorbic acid, glutathione, and carotenoids, as well as of antioxidative enzymes such as superoxide dismutase, ascorbate peroxidase (APX), and glutathione reductase. These systems scavenge ROS and regenerate the active forms of antioxidants (Zhu et al., 2013).

APX (EC 1.11.1.11) catalyzes the conversion of $\mathrm{H}_{2} \mathrm{O}_{2}$ to $\mathrm{H}_{2} \mathrm{O}$ and $\mathrm{O}_{2}$ using ascorbate as specific electron donor (Foyer and Halliwell, 1976). Thus, APX has a key function in scavenging ROS and in protection against toxic effects of ROS in higher plants (Asada, 1992). A relationship between salt tolerance and increased activation of APX has been demonstrated in Arabidopsis (Lu et al., 2007), tobacco (Liu et al., 2014), tomato (Wang et al., 2005), and other plants. Lu et al. (2007) also found that both of two cytosols (c) APXs (OsAPXa and OsAPXb) in rice can improve salt tolerance in transgenic Arabidopsis, but to a different extent, i.e. the $O s A P X b$ gene is more responsive to salt than the OsAPXa gene. Therefore, isolation and characterization of new cDNA of $A P X$ is utilized to further characterize the APX gene and provide new insights into the physiological roles and regulation of these enzymes by their overexpression in transgenic plants.

Recently, because of the high seed oil content and quality, low seed cost, rapid growth, adaptation to a wide range of agro-climatic conditions, and a bushy/shrub-like nature, Jatropha curcas has received special attention. It originates from arid and semiarid areas of South America, and it is distributed in all tropical regions. It grows in environments with unfavorable conditions, such as drought, salinity, and fluctuations in temperature, where most of the agriculturally important plant species are not able to thrive (Divakara et al., 2010). Previous studies have indicated that the antioxidant response might be one of the most important factors in J. curcas for the tolerance of abiotic stress (Gao et al., 2010). However, in contrast to other plants, the key antioxidative enzymes of $J$. curcas have not been well characterized at the molecular level.

In the present study, a novel APX gene was cloned from J. curcas by rapid amplification of cDNA end (RACE). The expression of $J c A P X$ was examined in different tissues and under salt stress conditions. Additionally, the gene was introduced into Arabidopsis by Agro- 
bacterium-mediated transformation to characterize the function of $J_{C A P X}$ under salt stress conditions. Subsequently, the differences between the wild type (WT) and transgenic plants were analyzed under $\mathrm{NaCl}$ stress conditions and optimal conditions. This research demonstrated that ectopic expression of the gene enhanced salt tolerance in transgenic Arabidopsis and suggested its possible application in improving the tolerance abilities in crops by the way of genetic modification.

\section{MATERIAL AND METHODS}

\section{Plant material and growth conditions}

Flowers, stems, leaves, and roots of $J$. curcas were gathered in the summer from Panzhihua city, Sichuan Province, China, and quickly frozen in liquid $\mathrm{N}_{2}$. The mature seeds were surface sterilized with $1 \% \mathrm{NaClO}$ for $10 \mathrm{~min}$ and washed with distilled water. Then their cotyledons were removed and placed in flasks containing Murashige and Skoog (MS) medium. Four days later, the rooted cotyledons were transferred to pots with a mixture of soil and vermiculite $(2: 1)$ in a chamber with photosynthetic active radiation of $200 \mu \mathrm{mol} \cdot \mathrm{m}^{-2} \cdot \mathrm{s}^{-1}$, a photoperiod of $16 \mathrm{~h} \mathrm{light} / 8 \mathrm{~h}$ dark, and temperature of $28^{\circ} \mathrm{C}$. The WT Arabidopsis thaliana seed (ecotype Col-0) were surface sterilized and plated on a solid MS medium. After three days of cold $\left(4^{\circ} \mathrm{C}\right)$ treatment, the plates were stored at $22^{\circ} \mathrm{C}$ in an incubator for propagation. The seedlings were transferred from the plates to a vermiculite-peat medium $(1: 1, \mathrm{v} / \mathrm{v})$ and grown to maturity at $22^{\circ} \mathrm{C}$ under 16 -h-light/8-h-dark cycle in a growth chamber.

\section{$J c A P X$ gene cloning and analysis}

For cloning of cDNA of $J c A P X$ from $J$. curcas, total RNA was isolated from young leaves using a TRIzol reagent (Invitrogen, Carlsbad, CA, USA). The cDNA was prepared from $1 \mu \mathrm{g}$ of total RNA using a SMART ${ }^{\mathrm{TM}} \mathrm{RACE} \mathrm{cDNA}$ Amplification kit (Clontech Laboratories Inc., Mountain View, CA, USA). A fragment of $J c A P X$ was amplified with two degenerate primers: JcAPX 1, 5'-ACATT(T/C)CTG(G/A)CTTCTG(C/A)TATCC-3' and JcAPX2, 5'-CTCG(G/A)CTG(C/T)G(T/G)TGG(T/A)ACTTCT-3', which were designed based on the conserved regions of the corresponding genes from other vascular plants. The 5'- and 3'-ends of $J c A P X$ were amplified by a BD SMART RACE cDNA Amplification Kit (Clontech Laboratories Inc.) as recommended by the manufacturer and corresponding primers. DNA sequencing was carried out on an ABI Prism 3730 DNA analyzer (Applied Biosystems, Foster City, CA, USA) at Huada Gene Laboratory (Shengzhen, China). Amino acid sequences were aligned with ClustalX (Larkin et al., 2007), and phylogenetic trees were constructed using the MEGA4 software (Tamura et al., 2011).

\section{Real-time PCR}

Total RNA was extracted from different tissues or leaves subjected to $200 \mathrm{mM} \mathrm{NaCl}$ stress with an RNA prep pure Plant kit (Tiangen Biotech Co., Ltd., Beijing, China). Each cDNA was synthesized using $1 \mu \mathrm{g}$ total RNA with a PrimeScript ${ }^{\mathrm{TM}}$ RT reagent Kit with gDNA Eraser (TaKaRa, Tokyo, Japan). Real time-PCR was performed on a CFX Connect ${ }^{\text {TM }}$ Real-Time PCR Detection System (Bio-RAD, Hercules, CA, USA) using an $\mathrm{iQ}^{\mathrm{TM}} \mathrm{SYBR}^{\circledR}$ Green Supermix 
(Bio-RAD). Data were obtained using the CFX Manager ${ }^{\mathrm{TM}}$ software (Bio-RAD). JcAPX fragment (156 bp) was amplified with gene-specific primers: JcAPX3, 5'-AGCTGTTATGTCGGCGTT CCT-3' and JcAPX4, 5'-GTTTCTCGGCTGCGTTGGTAC-3'. The $J$. curcas actin gene, 180bp long, which was amplified with primers Actin-F, 5'-ATGAGCTTCGAGTTGCACCA -3' and Actin-R, 5'-AGCATCAGTGAGATCACGAC-3', was used as a reference for normalizing the $J C A P X$ cDNA amounts.

\section{Plasmid construction and Arabidopsis transformation}

For JcAPX stable expression in Arabidopsis, the full length cDNA sequence was amplified and cloned into the BamHI-Sac I restriction enzyme sites of the pBI121 vector, in which the expression of $J c A P X$ was controlled by the CaMV $35 \mathrm{~S}$ promoter. Transformation of Arabidopsis was performed by the floral dip method using Agrobacterium tumefaciens strain EHA105 (Clough and Bent, 1998). T1 seeds were germinated on MS plates containing $50 \mathrm{mg} /$ $\mathrm{mL}$ kanamycin, and resistant plants were transferred to soil to obtain T2 seeds. Three independent lines of homozygous T3 plants containing a single insertion of each construct were used in the following analysis.

\section{Anti-salinity analysis}

For the germination salt tolerance assay, seeds were surface sterilized and sown on MS medium with or without the addition of $150 \mathrm{mM} \mathrm{NaCl}$ for 10 days under normal growth conditions $\left(22^{\circ} \mathrm{C}, 16\right.$-h-white light/8-h-dark cycle). Germinated seeds were cultured on MS medium with or without the addition of $125 \mathrm{mM} \mathrm{NaCl}$ under the same growth conditions to analyze the salt tolerance in seedlings.

\section{APX activity and $\mathrm{H}_{2} \mathrm{O}_{2}$ content assay}

APX activity was assayed according to Gest et al (2013). The reaction mixture (1.0 $\mathrm{mL}$ ) contained $50 \mathrm{mM}$ potassium phosphate buffer, $\mathrm{pH} 7.0,0.1 \mathrm{mM} \mathrm{H}_{2} \mathrm{O}_{2}, 0.5 \mathrm{mM}$ ascorbate, and $15 \mu \mathrm{L}$ protein extract. The reaction was initiated by the addition of $\mathrm{H}_{2} \mathrm{O}_{2}$, and ascorbate oxidation was measured at $290 \mathrm{~nm}$ for 1 min by using UV-VIS spectrophotometer (Hitachi U-2910, Tokyo, Japan). One unit of APX was defined as the amount of enzyme that oxidized 1 $\mu \mathrm{M}$ of ascorbate per min at $25^{\circ} \mathrm{C}$. Protein was determined by a Protein Assay Kit (Bio-RAD) using bovine serum albumin as a standard.

The histochemical detection of $\mathrm{H}_{2} \mathrm{O}_{2}$ in leaves was performed by in situ endogenous peroxidase-dependent staining. Whole explants of WT and transgenic Arabidopsis were submerged in $50 \mathrm{mM}$ Tris-acetate buffer ( $\mathrm{pH} 5.0$ ) with $0.1 \mathrm{mg} / \mathrm{mL} \mathrm{3,3-diamino-}$ benzidine (DAB) (Clemente-Moreno et al., 2012) and incubated at $25^{\circ} \mathrm{C}$ in the dark for 2 h. The leaves were then rinsed in $80 \%$ ethanol for $5 \mathrm{~min}$ at $60^{\circ} \mathrm{C}$ and photographed. For $\mathrm{H}_{2} \mathrm{O}_{2}$ content analyses, leaves $(0.5 \mathrm{~g})$ were homogenized with $2 \mathrm{~mL}$ Tris-acetate buffer $(50$ $\mathrm{mM}), \mathrm{pH} 5.0$, in the presence of $5 \mathrm{mM} \mathrm{KCN}$. The measurement of $\mathrm{H}_{2} \mathrm{O}_{2}$ was based on the peroxide-mediated oxidation of $\mathrm{Fe}^{2+}$, followed by the reaction of $\mathrm{Fe}^{3+}$ with xylenol orange (Bellicampi et al., 2000). 


\section{RESULTS}

\section{Cloning and characterization of $J c A P X$ gene}

Based on sequence homology among the APX genes or transcripts from other plants, the full-length cDNA (GenBank accession No.: KF792062) obtained by 5'- and 3'-RACE amplification consists of 1041 nucleotides and encodes a polypeptide of 346 amino acid residues with a calculated molecular mass of $37,810 \mathrm{Da}$ and theoretical isoelectric point of 8.40 . The alignment of the deduced amino acid sequence of $J c A P X$ with other $A P X \mathrm{~s}$ indicated that it was homologous to sequences from Vitis vinifera, Populus trichocarpa, and Citrus clementina. Additionally, highly conserved regions were in the $\mathrm{C}$-terminal end, whereas non-conserved region existed at the N-terminal end (Figure 1). Moreover, phylogenetic analysis of the amino acids of JcAPX group showed that JcAPX clustered with VvAPX from Vitis vinifera (Figure 2).

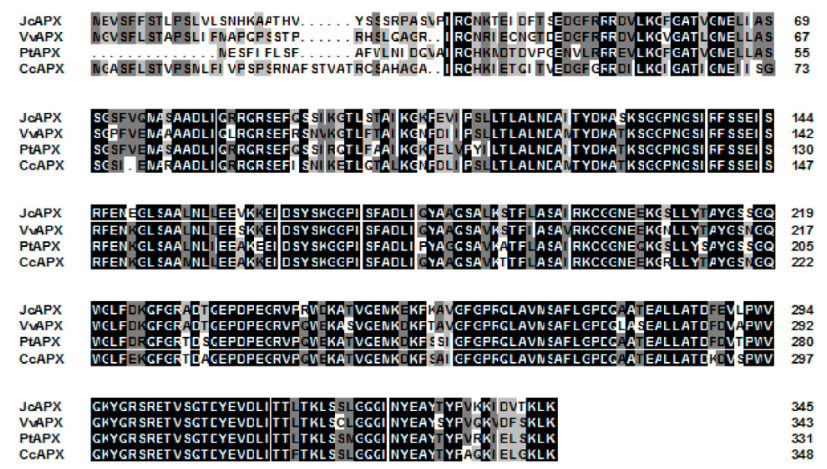

Figure 1. Alignment of the JcAPX sequence and its homologs using ClustalX. GenBank accession number and name of the species are as follows: JcAPX, Jatropha curcas APX (KF792062); VvAPX, Vitis vinifera APX (cbi19198.3); PtAPX, Populus trichocarpa APX (XP_002306565.1); CcAPX, Citrus clementina APX (XP_006433930). Identical amino acid residues are highlighted in black; conserved and semiconserved residues are highlighted in gray; dashes indicate gaps in the sequence introduced to optimize the alignment.

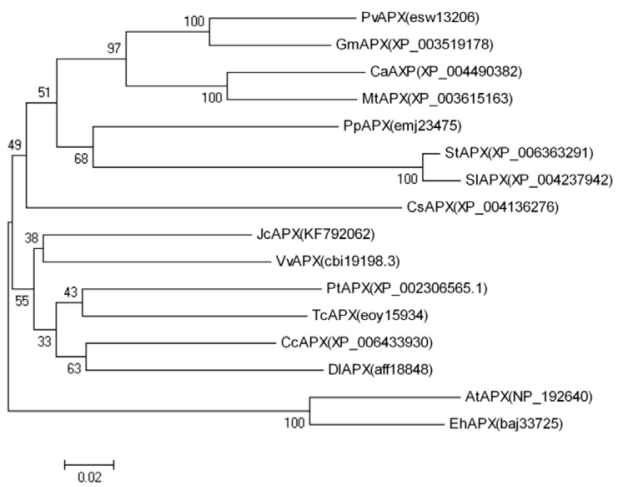

Figure 2. Phylogenetic tree based on amino acid sequences of APX inferred using the neighbor-joining method implemented in MEGA4. The sequences were obtained from GenBank and aligned with that of JcAPX. GenBank accession numbers are given in brackets. Pv, Phaseolus vulgaris; Gm, Glycine max; Ca, Cicer arietinum; Mt, Medicago truncatula; Pp, Prunus persica; St, Solanum tuberosum; Sl, Solanum lycopersicum; Cs, Cucumis sativus; $J c$, Jatropha curcas; Vv, Vitis vinifera; Pt, Populus trichocarpa; Tc, Theobroma cacao; Cc, Citrus clementina; Dl, Dimocarpus longan; At, Arabidopsis thaliana; Eh, Eutrema halophilum. 


\section{Comparison of mRNA expression levels of $J c A P X$}

The results of the spatial expression pattern tests indicated that $J c A P X$ was expressed in all $J$. curcas tissues, but the level of expression varied considerably among the tissues. The greatest level of expression of $J c A P X$ mRNA was in the leaf, and the lowest was in the stem (Figure 3A).

Prolonged treatment with $200 \mathrm{mM} \mathrm{NaCl}$ upregulated the $J c A P X$ 's expression in a time-dependent manner. The gene expression significantly increased after 6 h (Figure 3B), which is probably closely related to salt stress.
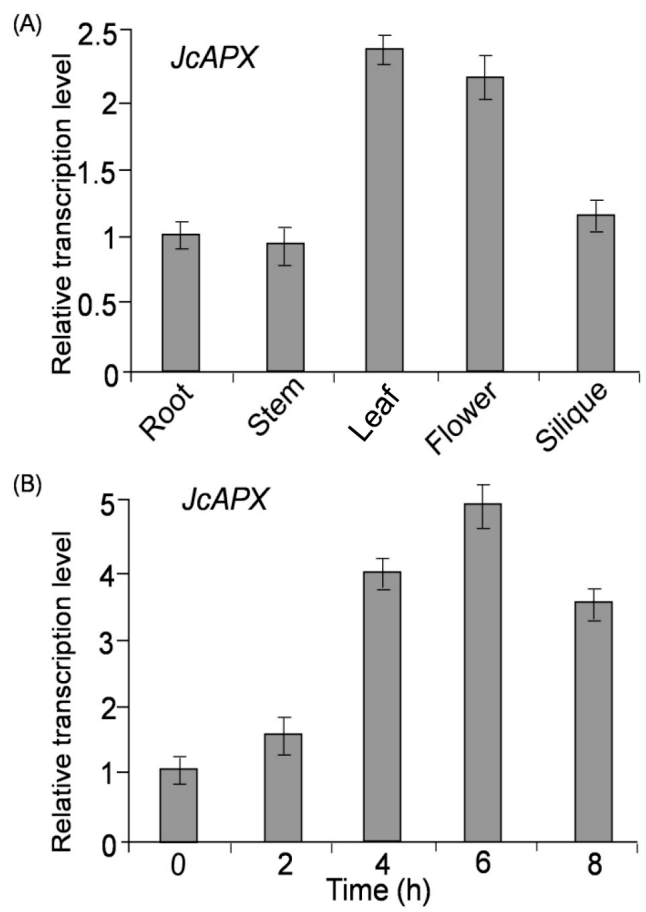

Figure 3. Expression pattern of $J c A P X$. (A) Real-time PCR analysis of $J c A P X$ gene expression in different plant organs. Total RNA was extracted from the root, stem, leaf, flower, and silique. Expression level of $J c A P X$ was monitored by real-time PCR against Actin. The level of expression in the root was set to 1 . The data are means of three separate runs and SDs are indicated. (B) qPCR analysis of JcAPX gene expression under salt stress conditions. Three-week-old seedlings were treated with $200 \mathrm{mM} \mathrm{NaCl}$. Total RNA was extracted at the indicated times and analyzed by qPCR. Actin was used as an internal control. The data are reported as means of three separate experiments and SDs are indicated.

\section{Molecular characterization of transgenic Arabidopsis plants}

The full-length $J c A P X$ sequence was placed under the control of CaMV-35S promoter. The resulting p35S: $J C A P X$ construct was successfully transformed into A. thaliana, and transgenic plants were detected with PCR after the first screening with $50 \mu \mathrm{g} / \mathrm{mL}$ kanamycin. Furthermore, the expression of the exogenous $J c A P X$ was confirmed by semi-quantitative real time-PCR. JcAPX transcripts were detected in transgenic lines T5, T12, and T19, which were chosen for further analysis (Figure 4). 


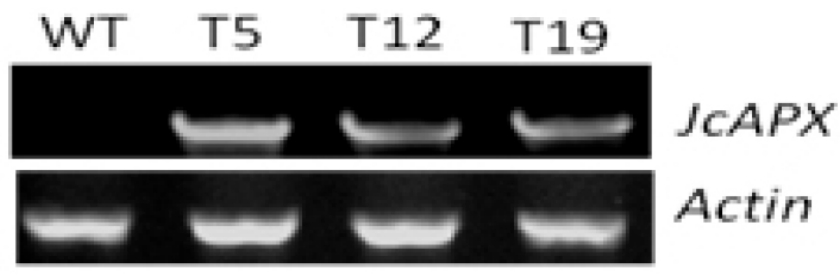

Figure 4. The transcript levels of $J c A P X$ in different overexpression lines were analyzed by real-time PCR. Total RNA was extracted from leaf samples collected from $T_{2}$ transgenic plants grown under normal conditions. The actin housekeeping gene was selected as an internal control.

\section{$J c A P X$ transgenic plants display tolerance to salt stress}

Seeds of three JcAPX-overexpressing lines and WT plants were germinated on MS plates supplemented with different concentration of $\mathrm{NaCl}$. Transgenic lines grown on MS plates without $\mathrm{NaCl}$ did not show any significant difference from the control group (Figure 5A and $\mathrm{C})$. However, the germination rates of $J_{C A P X}$-overexpressing lines sawn on the medium containing $150 \mathrm{mM} \mathrm{NaCl}$ were significantly higher than the germination rates in the WT (Figure $5 \mathrm{~B}$ and $\mathrm{D})$.

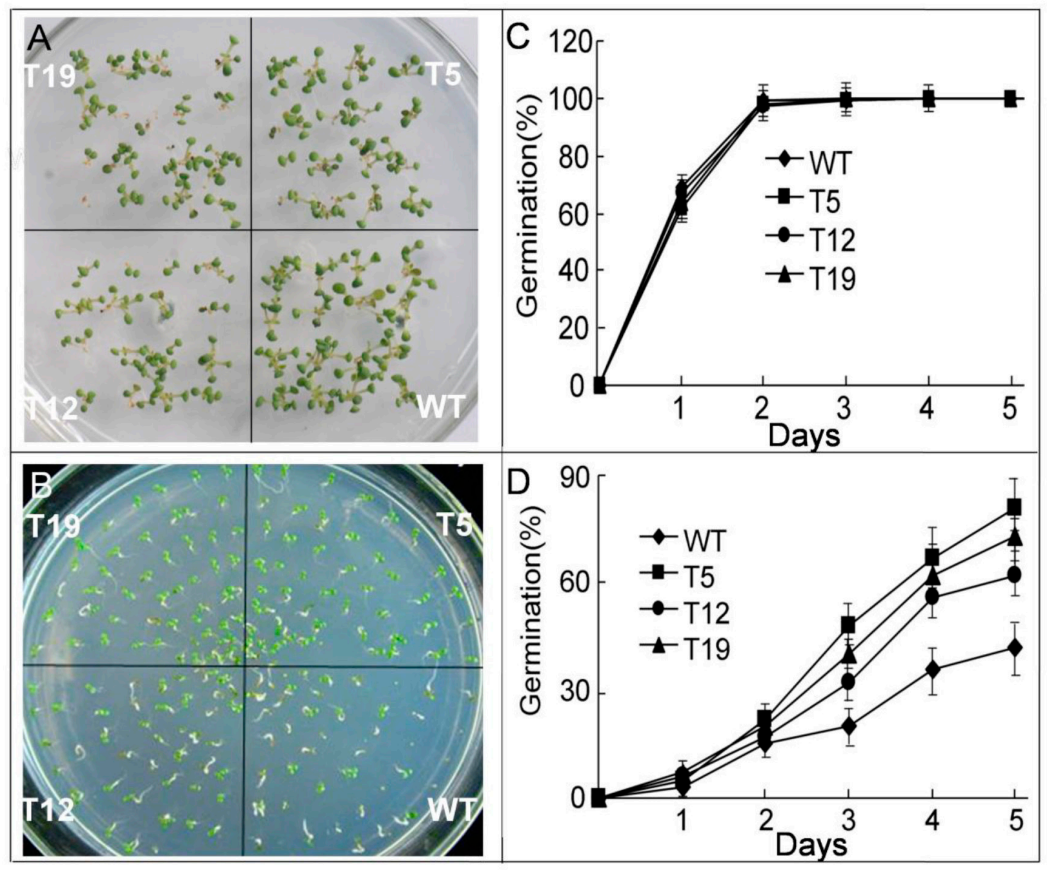

Figure 5. Salt stress response of wild-type and $J_{c} A P X$ transgenic plants during seed germination stage. Seeds were sown on MS medium without (A) and with $150 \mathrm{mM} \mathrm{NaCl}(\mathbf{B})$. Photos were taken five days after stratification. Percentage of germinating seeds grown for a period as indicated under normal condition (C) and $\mathrm{NaCl}$ treatment (D). Data are reported as means $\pm \mathrm{SD}(\mathrm{n}>100)$ from three independent experiments. WT, wild type; T5, T12, and $\mathrm{T} 19$, are independent $J c A P X$ transgenic lines. 
After a 5-day treatment with $150 \mathrm{mM} \mathrm{NaCl}$ on MS medium, the germination ratio was 78,82 , and $73 \%$ in the $\mathrm{T} 5, \mathrm{~T} 12$, and $\mathrm{T} 19 J_{c} A P X$ transgenic lines, respectively, while the ratio decreased to $36 \%$ in the untreated control groups. To further investigate the effects of high salinity on postgermination growth, root lengths were compared between the WT and transgenic seedlings under high salinity conditions. There were no obvious discrepancies in the morphology and growth characteristics when the plants were grown on normal MS medium (Figure $6 \mathrm{~A})$. However, in the presence of $150 \mathrm{mM} \mathrm{NaCl}$, the transgenic plants were more vigorous than the WT, forming longer primary root and more lateral roots (Figure 6B).

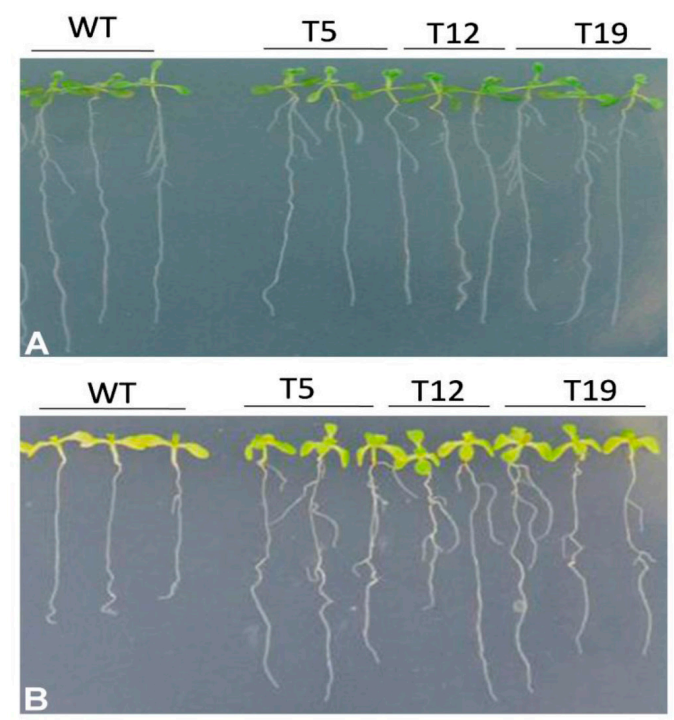

Figure 6. Transgenic plants are more tolerant than the wild-type plants to $\mathrm{NaCl}$ treatment. Root growth of germinated seedlings on MS without (A) and with $150 \mathrm{mM} \mathrm{NaCl}(\mathbf{B})$. After $48 \mathrm{~h}$ of stratification, seeds were grown on MS medium for 3 days, and then transferred to a medium containing $150 \mathrm{mM} \mathrm{NaCl}$ and growing vertically for additional 9 days. WT, wild type; T5, T12, and T19, are independent $J c A P X$ transgenic lines.

\section{APX activity and $\mathrm{H}_{2} \mathrm{O}_{2}$ levels under salt stress conditions}

To identify the influence of salt on APX activity, we investigated the APX activity in all lines tested. As shown in Figure 7, under controlled conditions, the APX activity in the three transgenic lines was higher than in the WT plants. When exposed to $150 \mathrm{mM} \mathrm{NaCl}$, the difference was more evident. After $24 \mathrm{~h}$ of treatment, the APX activity in transgenic lines increased about 0.9 times (T5), 0.4 times (T12), and 0.6 times compared with the APX activity in the WT (Figure 7).

Since the main function of APX is scavenging $\mathrm{H}_{2} \mathrm{O}_{2}$, it is necessary to detect the endogenous $\mathrm{H}_{2} \mathrm{O}_{2}$ content in transgenic plants and the WT. Therefore a histochemical staining with $\mathrm{DAB}$ was performed after the salt stress treatment. The results showed that, after the treatment with $150 \mathrm{mM} \mathrm{NaCl}$, only a weak and non-specific brownish staining was detected in transgenic lines T5 and T19. DAB staining in transgenic line T12 was visible only in the internodes, whereas this coloration in the WT was very intense in the entire seedling (Figure 8A). $\mathrm{H}_{2} \mathrm{O}_{2}$ content was also quantified in plant leaves. Lines T5, T12, and T19 exhibited approxi- 
mately 26,19 , and $37 \%$ lower $\mathrm{H}_{2} \mathrm{O}_{2}$ concentration, respectively, than the non-transformed control (Figure 8B). These results are consistent with those from DAB staining.

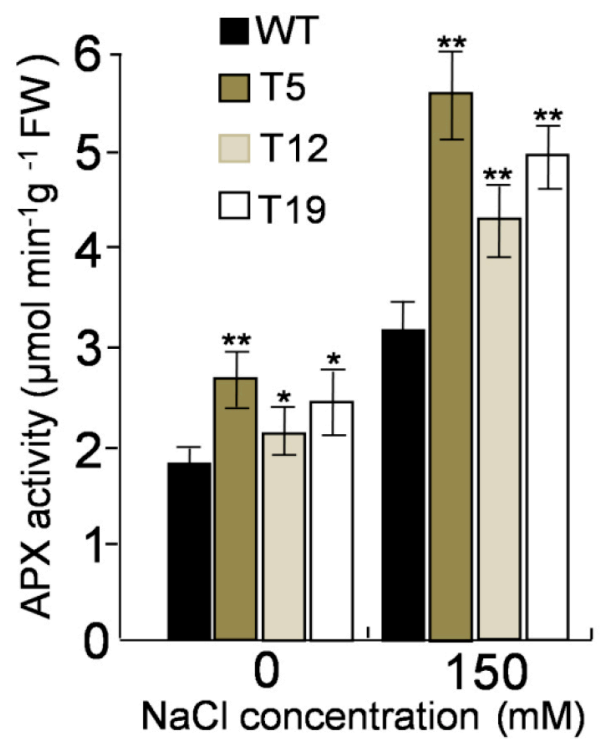

Figure 7. Activity assay of APX in transgenic and WT plants. Values are reported as means $(n=3) \pm S D$ of four independent experiments. * and ** represent significant differences determined by the Student $t$-test at $\mathrm{P}<0.05$, and $\mathrm{P}<0.01$, respectively.
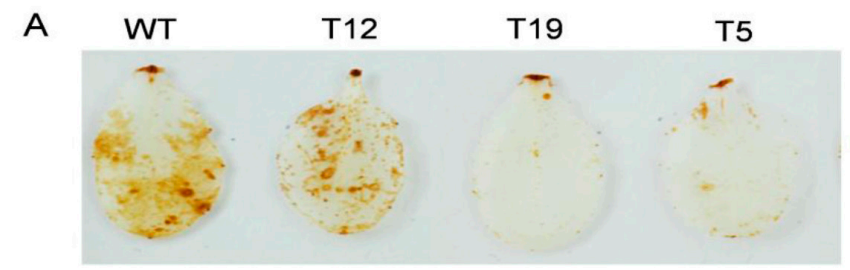

B

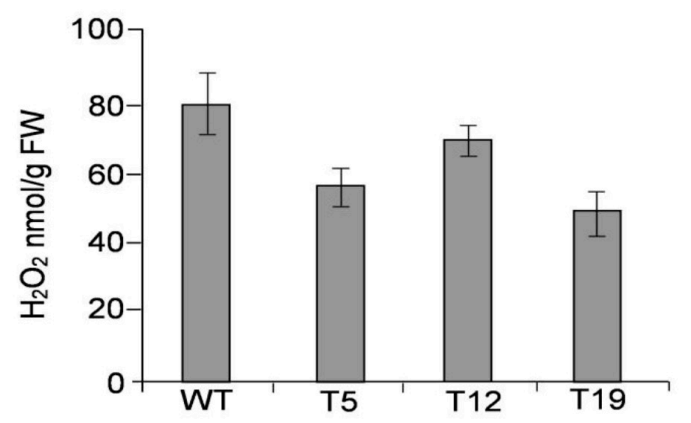

Figure 8. Effect of $J c A P X$ transformations on $\mathrm{H}_{2} \mathrm{O}_{2}$ content. A. Detection of $\mathrm{H}_{2} \mathrm{O}_{2}$ accumulation in DAB-stained Arabidopsis leaves. B. Endogenous $\mathrm{H}_{2} \mathrm{O}_{2}$ contents in leaves. Data are reported as means $(\mathrm{n}=3) \pm \mathrm{SD}$ of four independent experiments. 


\section{DISCUSSION}

APXs, especially the cytosolic APX, are thought to be involved in the protection of plants against oxidative stress conditions (Shigeoka et al., 2002). It has been reported that the overexpression of $A P X$ is essential for effective protection of plants against oxidative damage caused by salt stress (Diaz-Vivancos et al., 2013). In the present study, we isolated a novel APX gene from $J$. curcas and examined its potential function in transgenic Arabidopsis under salt stress conditions. Sequence alignment of JcAPX with other plants suggests almost identical function with other reported homologous proteins. Our present data indicate that $J C A P X$ is gradually and strongly induced by $\mathrm{NaCl}$ stress (Figure 3B). Similarly, the expression of $A P X$ genes from other species is upregulated under salt stress (Sun et al., 2010; Liu et al., 2014; Singh et al., 2014).

Although growth in all tested lines was suppressed under the salt stress, transgenic Arabidopsis lines showed significantly higher seed germination rate and evidently reduced sensitivity to $\mathrm{NaCl}$ in seedling stage under salt stress conditions (Figures 5 and 6). This was concurrent with previous reports of transgenic plants overexpressing the pepper APX or Рориlus pAPX genes (Sarowar et al., 2005; Sun et al., 2010; Wu et al., 2014). These results suggested that overexpression of JcAPX in transgenic lines with higher APX activity plays an effective role in alleviating this adverse stress conditions in Arabidopsis growth and development.

Whether under $\mathrm{NaCl}$ stress or not, APX activity was much higher in transgenic lines than in WT (Figure 7). High levels of APX activity can serve to directly scavenge $\mathrm{H}_{2} \mathrm{O}_{2}$ and maintain higher photosynthetic activity when plants are exposed to salt stress (Diaz-Vivancos et al., 2013). A notable symptom of oxidative damage is the marked accumulation of ROS such as $\mathrm{H}_{2} \mathrm{O}_{2}$ when plants are subjected to salt stress (Negrão et al., 2013). The accumulation of $\mathrm{H}_{2} \mathrm{O}_{2}$ due to salt stress has been reported in rice, marine macroalgae, and Medicago sativa (Luo and Liu, 2011; Hu et al., 2012; Mishra et al., 2013; Guo et al., 2014). Our results showed that WT plants accumulate large amounts of $\mathrm{H}_{2} \mathrm{O}_{2}$, far more than the transgenic plants under salt stress conditions (Figure 8). This phenomenon further confirmed that APX is a central component in the $\mathrm{H}_{2} \mathrm{O}_{2}$ scavenging mechanism. Furthermore, Davletova et al. (2005) also demonstrated that $\mathrm{H}_{2} \mathrm{O}_{2}$ levels increase in the absence of the cytosolic ascorbate peroxidase 1 (APX1).

In summary, the expression of $J c A P X$ conferred the transgenic Arabidopsis seedlings to salt tolerance contents. Moreover, plants transformed displayed a greater increase in APX activity than the control plants when exposed to $\mathrm{NaCl}$. Transgenic plants suppressed the accumulation of $\mathrm{H}_{2} \mathrm{O}_{2}$, providing evidence for a pivotal role of JcAPX in $\mathrm{H}_{2} \mathrm{O}_{2}$ scavenging. Therefore, $J C A P X$ is a potential candidate for improving abiotic stress tolerance and could be used to engineer salt stress tolerance in crop plants.

\section{ACKNOWLEDGMENTS}

Research funded by the National Natural Science Foundation of China (\#51109147 and \#51208322), Science and Technology Agency of Sichuan Province (\#2012JY0016), and the Strategic Emerging Industries of Guangdong Province (\#2012A020800003).

\section{REFERENCES}

Asada K (1992). Ascorbate peroxidase-a hydrogen peroxide-scavenging enzyme in plants. Physiol. Plant. 85: 235-241. Bellicampi D, Dipierro N, Salvi G, Cervone F, et al. (2000). Extracellular $\mathrm{H}_{2} \mathrm{O}_{2}$ induced by oligogalacturonides is not involved in the inhibition of the auxin-regulated roIB gene expression in tobacco leaf explants. Plant Physiol. 122: 1379-1386. 
Clemente-Moreno MJ, Diaz-Vivancos P, Piqueras A, and Hernández JA (2012). Plant growth stimulation in Prunus species plantlets by BTH or OTC treatments under in vitro conditions. J. Plant Physiol. 169: 1074-1083

Clough SJ and Bent AF (1998). Floral dip: a simplified method for Agrobacterium-mediated transformation of Arabidopsis thaliana. Plant J. 16: 735-743.

Davletova S, Rizhsky L, Liang H, Zhong SQ, et al. (2005). Cytosolic ascorbate peroxidase 1 is a central component of the reactive oxygen gene network of Arabidopsis. Plant Cell. 17: 268-281.

Diaz-Vivancos P, Faize M, Barba-Espin G, Faize L, et al. (2013). Ectopic expression of cytosolic superoxide dismutase and ascorbate peroxidase leads to salt stress tolerance in transgenic plums. Plant Biotechnol. J. 11: 976-985.

Divakara BN, Upadhyaya HD, Wani SP, Laxmipathi Gowda CL (2010). Biology and genetic improvement of Jatropha curcas L.: A review. Appl. Energy 87: 732-742.

Foyer CH and Halliwell B (1976). The presence of glutathione and glutathione reductase in chloroplasts: A proposed role in ascorbic acid metabolism. Planta, 133: 21-25.

Gao S, Ou-yang C, Tang L, Zhu J, et al. (2010). Growth and antioxidant responses in Jatropha curcas seedling exposed to mercury toxicity. J. Hazard Mater. 182: 591-597.

Gest N, Garchery C, Gautier H, Jiménez A, et al. (2013). Light-dependent regulation of ascorbate in tomato by a monodehydroascorbate reductase localized in peroxisomes and the cytosol. Plant Biotechnol. J. 11: 344-354.

Guo Z, Tan J, Zhuo C, Wang C, et al. (2014). Abscisic acid, $\mathrm{H}_{2} \mathrm{O}_{2}$ and nitric oxide interactions mediated cold-induced S-adenosylmethionine synthetase in Medicago sativa subsp. falcata that confers cold tolerance through up-regulating polyamine oxidation. Plant Biotechnol. J. 12: 601-612.

Hu L, Li H, Pang H and Fu J (2012). Responses of antioxidant gene, protein and enzymes to salinity stress in two genotypes of perennial ryegrass (Lolium perenne) differing in salt tolerance. J. Plant Physiol. 169: 146-156.

Imlay JA (2008). Cellular defenses against superoxide and hydrogen peroxide. Ann. Rev. Biochem. 77: 755-776.

Larkin MA, Blackshields G, Brown NP, Chenna R, et al. (2007). Clustal W and Clustal X version 2.0. Bioinformatics, 23: 2947-2948.

Liu Z, Bao H, Cai J, Han J, et al. (2014). A novel thylakoid ascorbate peroxidase from Jatropha curcas enhances salt tolerance in transgenic tobacco. Int. J. Mol. Sci. 15: 171-185.

Lu Z, Liu D and Liu S (2007). Two rice cytosolic ascorbate peroxidases differentially improve salt tolerance in transgenic Arabidopsis. Plant Cell Rep. 26: 1909-1917.

Luo MB and Liu F (2011). Salinity-induced oxidative stress and regulation of antioxidant defense system in the marine macroalga Ulva prolifera. J. Exp. Mar. Bio. Ecol. 409: 223-228.

Mishra P, Bhoomika K and Dubey RS (2011). Differential responses of antioxidative defense system to prolonged salinity stress in salt-tolerant and salt-sensitive India rice (Oryza sativa L.) seedlings. Protoplasma. 250: 3-19.

Negrão S, Almadanim MC, Pires IS, Abreu IA, et al. (2013). New allelic variants found in key rice salt-tolerance genes: an association study. Plant Biotechnol. J. 11: 87-100.

Sarowar S, Kim EN, Kim YJ, Ok SH, et al. (2005). Overexpression of a pepper ascorbate peroxidase-like 1 gene in tobacco plants enhances tolerance to oxidative stress and pathogens. Plant Sci. 169: 55-63.

Shigeoka S, Ishikawa T, Tamoi M, Miyagawa Y, et al. (2002). Regulation and function of ascorbate peroxidase isoenzymes. J. Exp. Bot. 53: 1305-1319.

Singh N, Mishra A and Jha B (2014). Over-expression of the peroxisomal ascorbate peroxidase (SbpAPX) gene cloned from halophyte Salicornia brachiata confers salt and drought stress tolerance in transgenic tobacco. Mar. Biotechnol. 16: 321-332.

Sun WH, Duan M, Shu DF, Yang S, et al. (2010). Over-expression of StAPX in tobacco improves seed germination and increases early seedling tolerance to salinity and osmotic stresses. Plant Cell Rep. 29: 917-926.

Tamura K, Peterson D, Peterson N, Stecher G, et al. (2011). MEGA5: Molecular evolutionary genetics analysis using maximum likelihood, evolutionary distance, and maximum parsimony methods. Mol. Biol. Evol. 28: 2731-2739.

Wang Y, Wisniewski M, Meilan R, Cui M, et al. (2005). Overexpression of cytosolic ascorbate peroxidase in tomato confers tolerance to chilling and salt stress. J. Amer. Soc. Hort. Sci. 130: 167-173.

Wu G, Wang G, Ji J, Gao H, et al. (2014). Cloning of a cytosolic ascorbate peroxidase gene from Lycium chinense Mill. and enhanced salt tolerance by overexpressing in tobacco. Gene 543: 85-92.

Zhu JK (2001). Plant salt tolerance. Trends Plant Sci. 6: 66-71.

Zhu C, Sanahuja G, Yuan D, Farré G, et al. (2013). Biofortification of plants with altered antioxidant content and composition: genetic engineering strategies. Plant Biotechnol. J. 11: 129-141. 